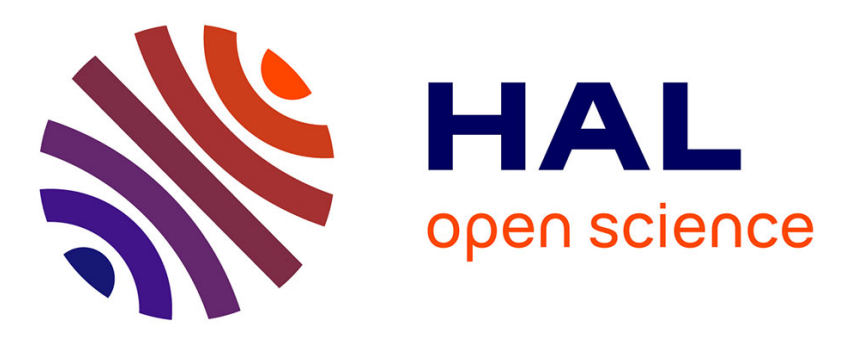

\title{
Les mesures de viscosité des liquides GexSe1-x dans les compositions a vitrification aisée et le concept d'association
}

\author{
G. Chaussemy, J. Fornazero, A. Laugier
}

\section{- To cite this version:}

G. Chaussemy, J. Fornazero, A. Laugier. Les mesures de viscosité des liquides GexSe1-x dans les compositions a vitrification aisée et le concept d'association. Revue de Physique Appliquée, 1977, 12 (5), pp.687-690. 10.1051/rphysap:01977001205068700 . jpa-00244226

\section{HAL Id: jpa-00244226 https://hal.science/jpa-00244226}

Submitted on 1 Jan 1977

HAL is a multi-disciplinary open access archive for the deposit and dissemination of scientific research documents, whether they are published or not. The documents may come from teaching and research institutions in France or abroad, or from public or private research centers.
L'archive ouverte pluridisciplinaire $\mathbf{H A L}$, est destinée au dépôt et à la diffusion de documents scientifiques de niveau recherche, publiés ou non, émanant des établissements d'enseignement et de recherche français ou étrangers, des laboratoires publics ou privés. 


\title{
LES MESURES DE VISCOSITÉ DES LIQUIDES Ge $\mathrm{Ge}_{1-x}$ DANS LES COMPOSITIONS A VITRIFICATION AISÉE ET LE CONCEPT D'ASSOCIATION
}

\author{
G. CHAUSSEMY, J. FORNAZERO \\ Laboratoire de Physique Electronique, Université Claude Bernard-Lyon I, \\ 43 Bd du 11-Novembre 1918, 69621 Villeurbanne Cedex, France \\ A. LAUGIER \\ Laboratoire de Physique de la Matière, \\ Bât. 502, I. N. S. A. de Lyon, 20 Avenue A.-Einstein, 69621 Villeurbanne Cedex, France
}

\begin{abstract}
Résumé. - Une corrélation est établie entre le diagramme de phase liquide-solide, la facilité de vitrification et la viscosité cinématique des liquides $\mathrm{Ge}_{x} \mathrm{Se}_{1-x}: 0 \leqslant x \leqslant 1 / 3$. On observe une augmentation de la viscosité avec $x$, attribuée à l'effet de branchement produit par Ge sur les chaînes de Se. Pour des compositions inférieures à celles de l'eutectique $(x=0,08)$, la variation avec la température est bien décrite par un modèle de polymère liquide dans le formalisme de volume libre, comme pour Se pur. Au-delà de l'eutectique, l'énergie d'activation augmente fortement avec $x$. Comme dans l'état vitreux, on suppose la présence de tétraèdres $\mathrm{GeSe}_{4}$. On propose une description utilisant le concept d'association de Jordan. Les modifications dans le comportement du liquide sont attribuées aux variations du nombre des espèces associées. Les variations isothermes de la viscosité avec $x$ sont bien décrites par le modèle. Celui-ci explique aussi l'allure du diagramme de phase. Entre l'eutectique et $\mathrm{GeSe}_{2}$, le liquidus théorique est en bon accord avec l'expérience.
\end{abstract}

\begin{abstract}
A correlation is established between the liquid-solid phase diagram, the easy glass formation and the kinematic viscosity of the liquids $\mathrm{Ge}_{x} \mathrm{Se}_{1-x}: 0 \leqslant x \leqslant 1 / 3$. The branching effect of $\mathrm{Ge}$ on Se chains explains the increase of the viscosity. For compositions minus than 0.08 (eutectic) a good description of the temperature variation is obtained by a polymer model in the free volume formalism, as for pure Se. After eutectic, the activation energy increases strongly with $x$. $\mathrm{GeSe}_{4}$ tetraedra are assumed, as for glasses. The Jordan's concept of association is used. The changes in the liquid properties are attributed to the changes in the number of associated species. A good description is obtained for the isotherm variations of viscosity and for the liquidus line of the phase diagram, in the composition range eutectic-GeSe $\mathrm{G}_{2}$.
\end{abstract}

1. Introduction. - Dans le système Ge-Se, le domaine de vitrification aisée est compris entre Se et $\mathrm{GeSe}_{2}$ [1-3]. Les compositions voisines de l'eutectique $(x=0,08)$ sont les plus favorables. Ce comportement se retrouve aussi sur les ternaires $\mathrm{GeSe}_{2}-\mathrm{Te}$ [4]. La viscosité est sensible aux modifications dans les arrangements atomiques. L'objectif de ce travail est de préciser la corrélation entre la viscosité, les particularités du diagramme de phase et la structure atomique dans ce domaine de composition.

Excepté pour le sélénium [5] la structure atomique n'a pas été déterminée directement. Le même type de structure que dans les amorphes peut être retenu, les liaisons restant covalentes $[6,7,8]$. Le diagramme de phase est connu [3,8]. Il est représenté sur la figure 1 . Le diagramme de phase liquide-solide des composés IIVI et IV-VI et de leurs solutions ternaires a été décrit avec succès [9] en utilisant le concept de solution associée de Jordan [10]. Les mesures de viscosité sont données dans la section 2. Le modèle de solution associée, dans le cas de $\mathrm{GeSe}_{2}$, est développée en section 3. Les variations de viscosité sont attribuées aux changements du nombre des espèces associées. Dans la section 4, le modèle est utilisé pour décrire le liquidus.

2. Résultats. - La viscosité cinématique des liquides a été déterminée à l'aide d'un viscosimètre à réservoir scellé oscillant du type Meyer-Schvidkovskii. La procédure expérimentale a été décrite en détail dans la référence [7]. Le germanium et le sélénium utilisés étaient de haute pureté $(99,999 \%$ ). Les résultats sont portés sur la figure 2 qui représente les isothermes de viscosité en fonction de la composition $x$. Une corrélation étroite avec les particularités du diagramme de phase apparaît. Trois zones sont caractéristiques :
A : une zone comprise entre le sélénium pur et l'eutec- tique,
B : la région de l'eutectique,
$\mathrm{C}:$ la zone comprise entre l'eutectique et $\mathrm{GeSe}_{2}$. 


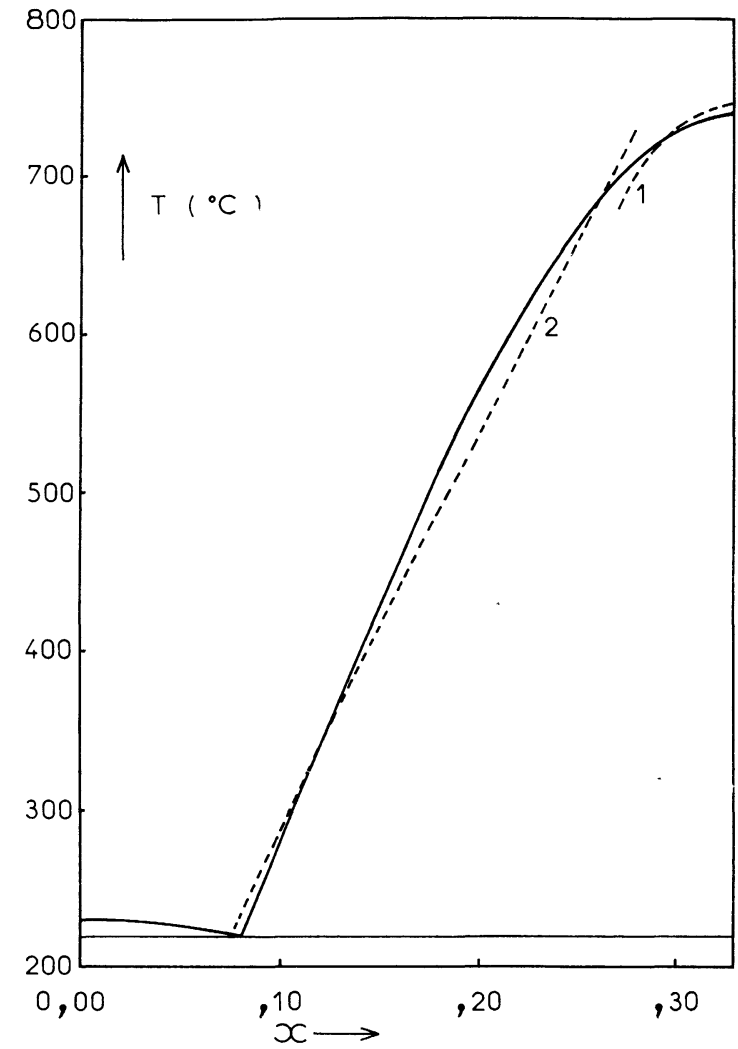

Fig. 1. - Diagramme de phase du système Ge-Se dans la zone de vitrification aisée (d'après les références [3 et 8]). La ligne continue est le liquidus expérimental. La courbe en tirets 1 est le liquidus calculé par la relation approchée (11). La courbe en tirets 2 est le liquidus calculé par la relation (10).

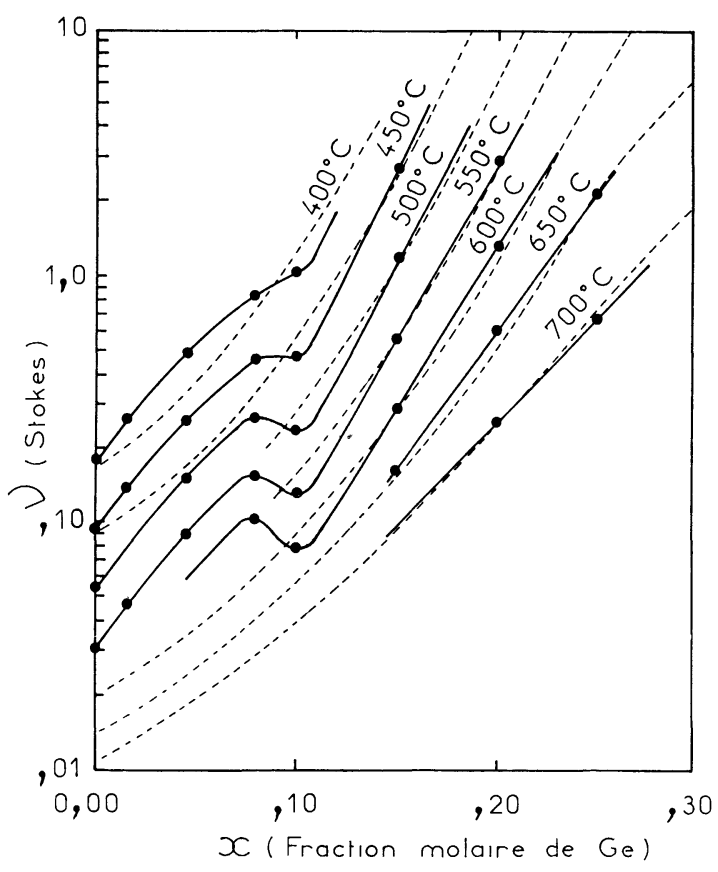

FIG. 2. - Isothermes de viscosité des liquides $\mathbf{G e}_{x} \mathrm{Se}_{2-x}$. Courbes continues : expérience; courbes en tirets: théorie (liquide associé).
Dans la première zone, le liquide se comporte comme le sélénium pur. La relation suivante a été vérifiée dans un précédent travail [11]:

$$
v=\frac{B}{d} T^{1 / 2} \exp \left[\frac{E}{k T}+\frac{\pi}{12} \frac{a^{3}}{\alpha V\left(T-T_{\mathrm{g}}\right)}\right] .
$$

Cette relation est déduite de celle obtenue par Rialland et Perron [12] dans le cadre du formalisme du volume libre pour analyser la viscosité de Se pur et des alliages Se-Te. Dans cette relation, $\alpha$ est le coefficient de dilatation, $V$ le volume moléculaire moyen, $T_{\mathrm{g}}$ la température de vitrification, $a$ la distance des plus proches voisins, $d$ la densité. $B$ est une constante ajustable et $E$ l'énergie associée à la polymérisation du sélénium :

$$
n \mathrm{Se} \rightleftarrows\left[\mathrm{Se}_{n}\right], \quad \mathrm{Se}_{n}=[\mathrm{Se}]^{n} \exp E / k T .
$$

Les valeurs de $E$ sont portées sur la figure 3. $E$ diminue avec $x$ jusqu'à l'eutectique, mais croît très rapidement

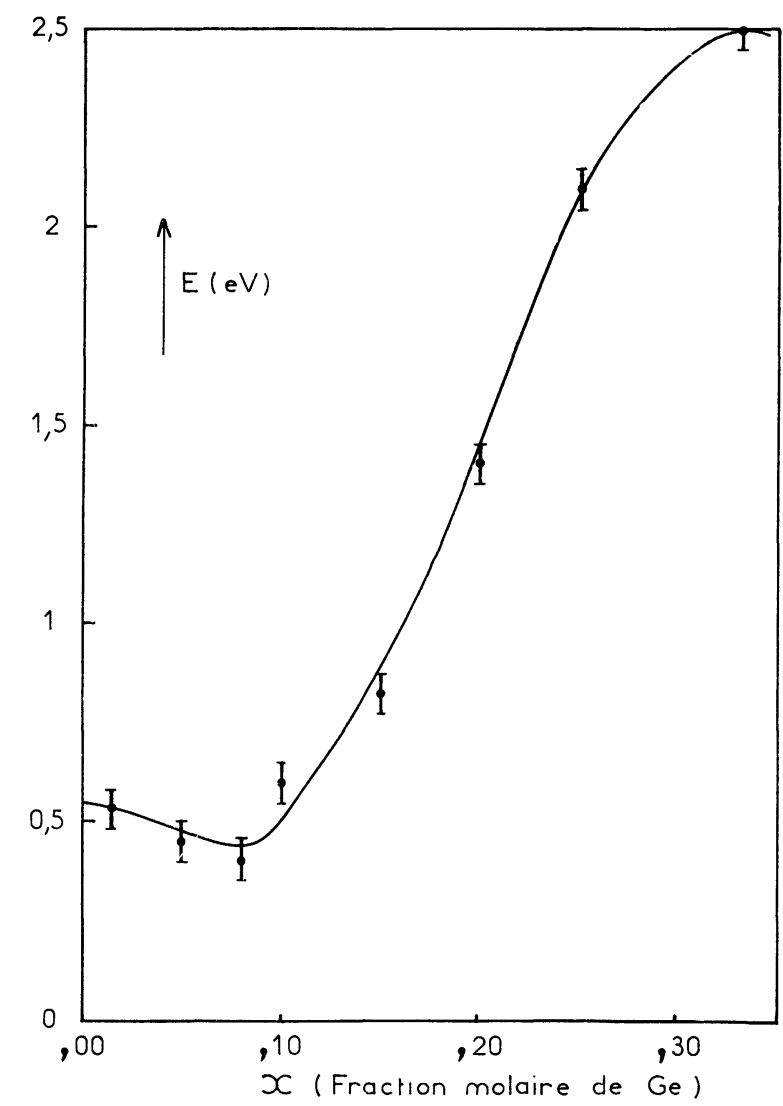

Fig. 3. - Energie d'activation de la viscosité. (L'erreur est $\pm 0,05 \mathrm{eV}$.) Noter la corrélation avec le diagramme de phase (Fig. 1).

au-delà. Dans cette première zone, l'introduction de germanium augmente l'interaction entre chaînes et donc, la viscosité. Si des anneaux $\mathrm{Se}_{8}$ sont présents, ils tendent alors à disparaître. Considérant $\alpha$ chaînes et $(1-\alpha)$ anneaux, l'équation (2) doit être modifiée :

$$
n^{\prime} \mathrm{Se} \rightleftarrows \alpha \mathrm{Se}_{n}+(1-\alpha) \mathrm{Se}_{8}
$$


et conduit à la valeur $E / \alpha$ pour l'énergie d'activation. Alors, supposant tous les anneaux disparus $(\alpha=1)$ à l'eutectique à cause du changement de structure du liquide, on peut déduire la concentration relative d'anneaux dans le sélénium pur, des valeurs de $E$ pour $x=0$ et $x=0,08$. On obtient $1-\alpha=0,27$. Par dissolution sélective dans $\mathrm{CS}_{2}$ de Se amorphe trempé à partir du liquide à $450 \mathrm{C}$, on a 0,26 . Cet accord peut, cependant, être fortuit et ne permet pas de conclure définitivement.

Au-delà de l'eutectique, la loi d'Arrhénius est bien suivie. Les énergies d'activation $E$ sont portées figure 3. Une corrélation étroite avec le liquidus apparaît.

3. Viscosité et liquide associé. - Dans la zone B apparaît une inflexion sur les isothermes de viscosité (Fig. 2). Glazov et al. [13] ont discuté les anomalies des diagrammes viscosité-composition pour les systèmes eutectiques. Pour le système $\mathrm{Se}-\mathrm{GeSe}_{2}$, les relations entre la viscosité et la structure du liquide ont déjà été discutées [7]. Soit $F_{i j}$ les forces de liaisons entre atomes. Les inflexions de la figure 2 indiquent que :

$$
F_{\mathrm{Se}-\mathrm{Se}}>F_{\mathrm{Ge}-\mathrm{Se}}>F_{\mathrm{Ge}-\mathrm{Ge}}
$$

pour les liquides riches en sélénium et que

$$
F_{\mathrm{Ge}-\mathrm{Se}}>F_{\mathrm{Se}-\mathrm{Se}}, \quad F_{\mathrm{Ge}-\mathrm{Se}}>F_{\mathrm{Ge}-\mathrm{Ge}}
$$

pour les liquides au-delà de l'eutectique. Dans cette zone, les liaisons entre atomes différents sont plus probables que les liaisons entre atomes identiques. Le concept d'association semble donc adapté à la description $\mathrm{du}$ comportement des liquides $\mathrm{Ge}-\mathrm{Se}$ dans cette zone.

Comme les amorphes correspondants, les liquides sont constitués de tétraèdres $\mathrm{GeSe}_{4}$ reliés par des atomes de Se [7]. La viscosité augmente avec la quantité de tétraèdres. Pour évaluer cette quantité en fonction de $x$, on considère la réaction d'association globale :

$$
\mathrm{Ge}+2 \mathrm{Se} \rightleftarrows \mathrm{GeSe}_{2}
$$

Cette réaction n'implique pas l'existence des molécules dans le liquide, mais traduit la condition

$$
F_{\mathrm{Ge}-\mathrm{Se}}>F_{\mathrm{Se}-\mathrm{Se}}
$$

et les restrictions sur les coordinances : 4 liaisons Ge-Se pour le germanium et 2 liaisons pour le sélénium. Les molécules sont seulement des concepts thermodynamiques permettant une description plus commode, comme il l'a été récemment discuté par Jordan et Ilegems [14]. Alors, $x_{i}$ étant la fraction molaire de l'espèce $i$ :

$$
\begin{aligned}
x_{\mathrm{Ge}} & =x\left(1-2 x_{\mathrm{GeSe}_{2}}\right)-x_{\mathrm{GeSe}_{2}} \\
x_{\mathrm{Se}} & =1-x\left(1+2 x_{\mathrm{GeSe}_{2}}\right) .
\end{aligned}
$$

La constante d'équilibre, $K(T)$, de la réaction (3) est :

$$
K=\frac{x_{\mathrm{Ge}} x_{\mathrm{Se}}^{2}}{s_{\mathrm{GeSe}_{2}}}
$$

$K=0$ correspond à une solution complètement associée, $K=\infty$ à une solution complètement dissociée (c'est le cas à haute température). Les valeurs de $x_{\mathrm{Ge}}, x_{\mathrm{Se}}, x_{\mathrm{GeSe}_{2}}$ sont obtenues en résolvant les équations (4) et (5) avec $K$ comme paramètre. Pour les solutions associées $(K<0,1), x_{\mathrm{Ge}}$ est négligeable, ce qui est normal.

La viscosité peut être déduite de ce calcul en utilisant la relation d'additivité suivante :

$$
\log \eta=\sum_{i} x_{i} \log \eta_{i}
$$

Cette relation a été vérifiée par Glazov dans l'étude de la cinétique des réactions chimiques [13]. $\log \eta_{\mathrm{Se}}$ est connu puisque à $x=0, x_{\mathrm{GeSe}_{2}}=0$. Cependant, sauf si $K=0$, les données expérimentales ne fournissent pas $\eta_{\mathrm{GeSe}_{2}}$ pour $x=1 / 3$. Des chaînes peuvent subsister. Il faut donc deux paramètres pour obtenir les courbes de viscosité isothermes : $K$ et $\eta_{\mathrm{GeSe}_{2}}$. Dans la zone $0,08 \leqslant x \leqslant 0,33$, un bon accord avec l'expérience est observé. Les valeurs de $K$ nécessaires sont $6 \times 10^{-2}$ à $700^{\circ} \mathrm{C}, 4 \times 10^{-2}$ à $650^{\circ} \mathrm{C}, 10^{-2}$ à $600^{\circ} \mathrm{C}$, de l'ordre de $10^{-3}$ en dessous de $500^{\circ} \mathrm{C}$. Ceci est normal. A haute température, le liquide se dissocie et $K$ augmente. Ceci explique pourquoi le comportement décrit dans ce travail n'a pas été observé par Glazov et al. [6] leurs mesures étant effectuées à des températures supérieures à $900^{\circ} \mathrm{C}$.

4. Association et diagramme de phase. - Le modèle proposé doit aussi permettre la description du liquidus entre l'eutectique et $\mathrm{GeSe}_{2}$. L'équation générale est $[9$, 14] :

$$
1=\frac{a}{a^{*}} \exp \frac{\Delta S}{R}\left(\frac{T_{\mathrm{F}}}{T}-1\right) .
$$

Dans cette relation $\Delta S$ et $T_{\mathrm{F}}$ sont l'entropie et la température de fusion de $\mathrm{GeSe}_{2}$. $a$ et $a^{*}$ sont les activités de $\mathrm{GeSe}_{2}$ dans le liquidus, $a^{*}$ correspond au liquide stœchiométrique.

En l'absence de données thermodynamiques sur les activités dans ce système, on va utiliser un modèle simplifié permettant d'obtenir une forme approchée du liquidus. Le calcul est directement déduit de celui utilisé par Jordan pour ZnTe et CdTe [10]. La différence provient de la forme plus compliquée de l'espèce associée du type $\mathrm{AB}_{n}$.

On définit le coefficient de dissociation :

$$
d=\left(x_{\mathrm{Ge}}+x_{\mathrm{Se}}\right) /\left(x_{\mathrm{Ge}}+x_{\mathrm{Se}}+3 x_{\mathrm{GeSe}_{2}}\right)
$$

et sa valeur particulière $\beta$ pour le liquide stœchiométrique. La constante $K$ (équation 5 ) ne dépend que de $T$ :

$$
K=\frac{4 \beta^{3}}{(1-\beta)(1+2 \beta)^{2}}=\frac{(d-1+3 x)(1+2 d-3 x)}{(1-d)(1+2 d)^{2}}
$$


Conservant le formalisme des solutions régulières pour Ge et Se, le coefficient d'activité $\gamma$ de $\mathrm{GeSe}_{2}$ est obtenu en intégrant l'équation de Gibbs-Duhem :

$$
\log \frac{\gamma_{\mathrm{GeSe}_{2}}}{\gamma_{\mathrm{GeSe}_{2}}^{*}}=\frac{\alpha}{3 R T}[1-3 x(2-3 x)]
$$

Le paramètre $\alpha$ s'identifie au paramètre d'interaction Ge-Se si la solution est dissociée et au paramètre $\mathrm{Se}-\mathrm{GeSe}_{2}$ si la solution est associée. Finalement, pour les solutions très associées, on obtient l'équation approchée du liquidus :

$$
\begin{array}{r}
1=\frac{(1+2 \beta)}{1-\beta} \frac{x}{1-2 x} \exp \left\{\frac{\Delta S}{R}\left(\frac{T_{\mathrm{F}}}{T}-1\right)+\right. \\
\left.+\frac{\alpha}{3 R T}[1-3 x(2-3 x)]\right\} .
\end{array}
$$

Cette équation est analogue à l'équation approchée obtenue par Jordan. A cause des approximations utilisées $(\beta$ indépendant de $x$ et de $T$ ), elle n'est pas valable au voisinage du point de fusion [10]. Cependant, une forme simple peut être obtenue pour cette région. Posant $y=x-1 / 3$, l'équation (8) donne :

$$
d=\beta+3 y^{2} / \beta
$$

et l'équation (7) se ramène à :

$$
T_{\mathrm{F}}-T=\frac{R T_{\mathrm{F}}}{\Delta_{S}} \cdot \frac{9 y^{2}}{\beta} .
$$

Les données utilisées par le calcul sont :

$$
\begin{array}{r}
T_{\mathrm{F}}=1019 \mathrm{~K} ; \Delta S=8 \text { u. e. } ; \quad \beta=0,15 ; \\
\alpha=-10,5 \mathrm{k} . \mathrm{cal} / \mathrm{mole} .
\end{array}
$$

La figure 1 montre les courbes calculées avec les équations (11) et (10). Compte tenu des approximations utilisées, l'accord est convenable.

5. Discussion. - Le concept d'association donne une description correcte du nombre respectif des entités présentes dans le liquide. Celui-ci est supposé idéal pour la viscosité alors que le liquidus (10) fait intervenir une solution régulière. Ces deux points ne sont pas contradictoires.

Au-dessus du liquidus, le terme d'entropie (qui est l'entropie de mélange idéal) dans l'expression de l'énergie de mélange, devient déterminant, ce qui justifie l'approximation. La valeur de $\beta$ utilisée dans l'équation (8) conduit à une valeur de $K$ égale à $9 \times 10^{-3}$, valeur cohérente avec celle déduite des mesures de viscosité.

En conclusion, dans la zone de vitrification aisée, le germanium augmente la viscosité (effet de branchement) et favorise l'association dans le liquide sous forme de tétraèdres $\mathrm{GeSe}_{4}$ reliés par des atomes de Se. La quantité de chaînes et d'anneaux diminue rapidement quand la quantité de germanium augmente. Une corrélation est établie entre le diagramme de phase et la viscosité en liaison avec les changements structurels dans le liquide. L'étude de Laurant et Ribeyron [15] sur la structure des amorphes par les spectres d'absorption I. R. confirme aussi cette corrélation.

\section{Bibliographie}

[1] Feltz, A., Buttner, H. J., Lippmann, F. J., Maul, W., J. Non-Crystall. Solids 8-10 (1972) 64.

[2] Lochman, R. E., Armstrong, A. J., Forestone, D. W., Gould, R. W., J. Non-Crystall. Solids 8-10 (1972) 72.

[3] Azoulay, R., Thibierge, H., Brenac, A., J. Non-Crystall. Solids 18 (1975) 33.

[4] Bordas, S., Clavaguera, N., Baro, M. D., Casas-VazQuez, J., Clavaguera-Mora, M. T., Revue Phys. Appl. 12 (1977) 681.

[5] Tourand, G., J. Physique 34 (1973) 937.

[6] Glazov, V. M., Situlina, O. V., Kok. Akad. Nauk, SSSR, 187 (1969) 799.

[7] Laugier, A., Chaussemy, G., Fornazero, J., J. Non-Cryst. Solids (1977) à paraître.
[8] Bensoussan, M., Revue Phys. Appl. 12 (1977) 753.

[9] LAUgier, A., Revue Phys. Appl. 8 (1973) 259.

[10] JoRdAN, A. S., Metall. Trans. 1 (1970) 239.

[11] Chaussemy, G., Fornazero, J., Laugier, A., J. Physique 37 (1976) L-238.

[12] Rialland, J. F., Perron, J. C., Proc. 6th Int. Conf. Amorphous and Liquid Semiconductors. Structure and Properties. Kolomiets ed. (1976) 371.

[13] Glazov, V. M., Chizhevskaya, S. N., Glazoleva, N. N., Liquid Semiconductors (Plenum Press, N. Y.) 1969.

[14] Jordan, A. S., Ilegems, M., J. Phys. Chem. Sol., 35 (1975) 329.

[15] Laurant, J. M., Ribeyron, A., Revue Phys. Appl. 12 (1977). 